\title{
APRENDIZAGEM DE ANATOMIA VERTEBRAL HUMANA POR MEIO DO USO DE MODELOS VERTEBRAIS LOMBARES 2D E 3D
}

\author{
Cristiane de Bona - Instituto Federal de Educação, Ciência e Tecnologia de Santa \\ Catarina, cris.debona19@gmail.com \\ Tatiane Sabriela Camozzato - Docente do Instituto Federal de Educação, Ciência e \\ Tecnologia de Santa Catarina, tatiane@ifsc.edu.br \\ Charlene da Silva - Docente do Instituto Federal de Educação, Ciência e Tecnologia \\ de Santa Catarina, charlene.silva@ifsc.edu.br \\ Patrícia Fernanda Dorow - Docente do Instituto Federal de Educação, Ciência e \\ Tecnologia de Santa Catarina, patriciad@ifsc.edu.br \\ Matheus Savi Brum, Docente do Instituto Federal de Educação, Ciência e Tecnologia \\ de Santa Catarina, matheus.savi@ifsc.edu.br
}

Resumo: Na área da saúde a tecnologia de prototipagem rápida possibilita a reprodução de modelos anatômicos fidedignos à estrutura original. Assim, o objetivo do estudo é comparar o desempenho avaliativo dos discentes após o uso de dois recursos didáticos distintos: desenhos esquemáticos (2D) da vértebra lombar e o modelo vertebral lombar em 3D confeccionado por prototipagem (impressão 3D). O estudo tem caráter exploratório. Participaram da pesquisa 29 discentes matriculados na disciplina de anatomia, estes utilizaram o desenhos em 2D e o modelo 3D como recurso didático e posteriormente realizaram uma avaliação prática por meio de um questionário semiestruturado. Não houve diferença impactante ao comparar o desempenho de ambos os recursos didáticos. Alguns acidentes ósseos, como pedículo e processo articular apresentaram acertos inferiores nos dois recursos. Conclui-se que os desenhos esquemáticos em 2D e o modelo impresso em 3D da vértebra lombar são ferramentas complementares no processo de ensino aprendizagem.

Palavras-chave: Modelos anatômicos. Impressão tridimensional. Anatomia. Aprendizagem.

\section{LEARNING HUMAN VERTEBRAL ANATOMY THROUGH THE USE OF 2D AND 3D LUMBAR VERTEBRAL MODELS}

\begin{abstract}
In the area of health, rapid prototyping technology enables the reproduction of anatomical models that are reliable to the original structure. Thus, the aim of the study is to compare the evaluative performance of students after the use of two distinct teaching resources: schematic (2D) drawings of the lumbar vertebra and the 3D lumbar vertebral model made by prototyping (3D printing). The study has an exploratory character. Twenty-nine students enrolled in the anatomy discipline participated in the survey, they used 2D drawings and 3D model as a didactic resource and later carried out a practical evaluation by means of a semi-structured questionnaire. There was no striking difference when comparing the performance of both teaching resources. Some bone accidents, such as pedicle and joint process, presented inferior hits in both resources. It is concluded that $2 \mathrm{D}$ schematic drawings and the 3D printed model of the lumbar vertebra are complementary tools in the teaching process.
\end{abstract}

Key-words: Anatomic models. Tridimensional printing. Anatomy. Learning. 


\section{Introdução}

A área da saúde é influenciada pelo avanço tecnológico, um exemplo é a utilização do recurso de prototipagem rápida, a qual permite a reprodução de estruturas do corpo humano em modelos impressos, com características fidedignas (Silva; Malagon, 2016).

O objeto resultante da prototipagem são nomeados biomodelos, estes são utilizados como ferramentas auxiliadoras no processo ensino da anatomia (Grande Neto, 2016). Sabe-se que um conhecimento sólido acerca de anatomia humana é fundamental para a atuação nas diversas áreas da saúde, em vista disto torna-se importante a reflexão quanto à aplicação de recursos tecnológicos no desenvolvimento de metodologias que auxiliem efetivamente no processo ensino aprendizagem dos discentes (Estai e Bunt, 2016).

A prototipagem rápida existe desde a década de 80 , contudo a diminuição dos custos de equipamentos e insumos levou a uma grande expansão dessa tecnologia, tornando-a mais acessível também ao meio educacional e passível de proporcionar ao estudante a noção tridimensional, contribuindo assim, na compreensão das diferentes estruturas do corpo humano (Nishimura, 2016).

Nesta perspectiva, os discentes deste século, fazem parte de uma geração denominada "nativos digitais", que, diferentemente da geração anterior, os "imigrantes digitais", nasceram em uma época dominada pelas tecnologias de informação e comunicação, apresentando diferenças na forma de acessar e processar informações (Corredera, 2014). Dessa forma, entende-se que é fundamental proporcionar aos acadêmicos, recursos didáticos compatíveis à sua realidade, explorando os benefícios oferecidos pelo constante avanço tecnológico (Paiva et al., 2013).

Portanto, o uso da prototipagem rápida no ensino de anatomia pode ser uma boa alternativa, tendo em vista que, esse tipo de modelo reproduz tridimensionalmente as características da peça anatômica cadavérica. Isso pode permitir ao aluno uma experiência de aprendizado mais efetiva, além de ser uma ferramenta eventualmente confeccionada pela própria instituição de ensino (Matozinhos, 2017).

Nesse contexto, em busca de um novo recurso de ensino prático que possa ser aplicado na disciplina de Anatomia, o estudo pretende comparar o desempenho avaliativo dos discentes após o uso de dois recursos didáticos distintos: desenhos esquemáticos (2D) da vértebra lombar e o modelo vertebral lombar em confeccionado por prototipagem (impressão 3D).

\section{Material e Método}

A pesquisa tem cunho exploratório, sendo que foi realizada em uma instituição federal de ensino que oferece um curso de graduação na área da saúde. No projeto pedagógico do referido curso há a disciplina de anatomia. Participaram da pesquisa 29 discentes matriculados na referida disciplina, que foram divididos em dois grupos, denominados Turma A (15 participantes) e Turma B (14 participantes), conforme o plano de ensino. A operacionalização do estudo ocorreu segundo ilustra na Figura 1.

V. $18 \mathrm{~N}^{\mathrm{o}} 1$, julho, 2020 RENOTE DOI: 


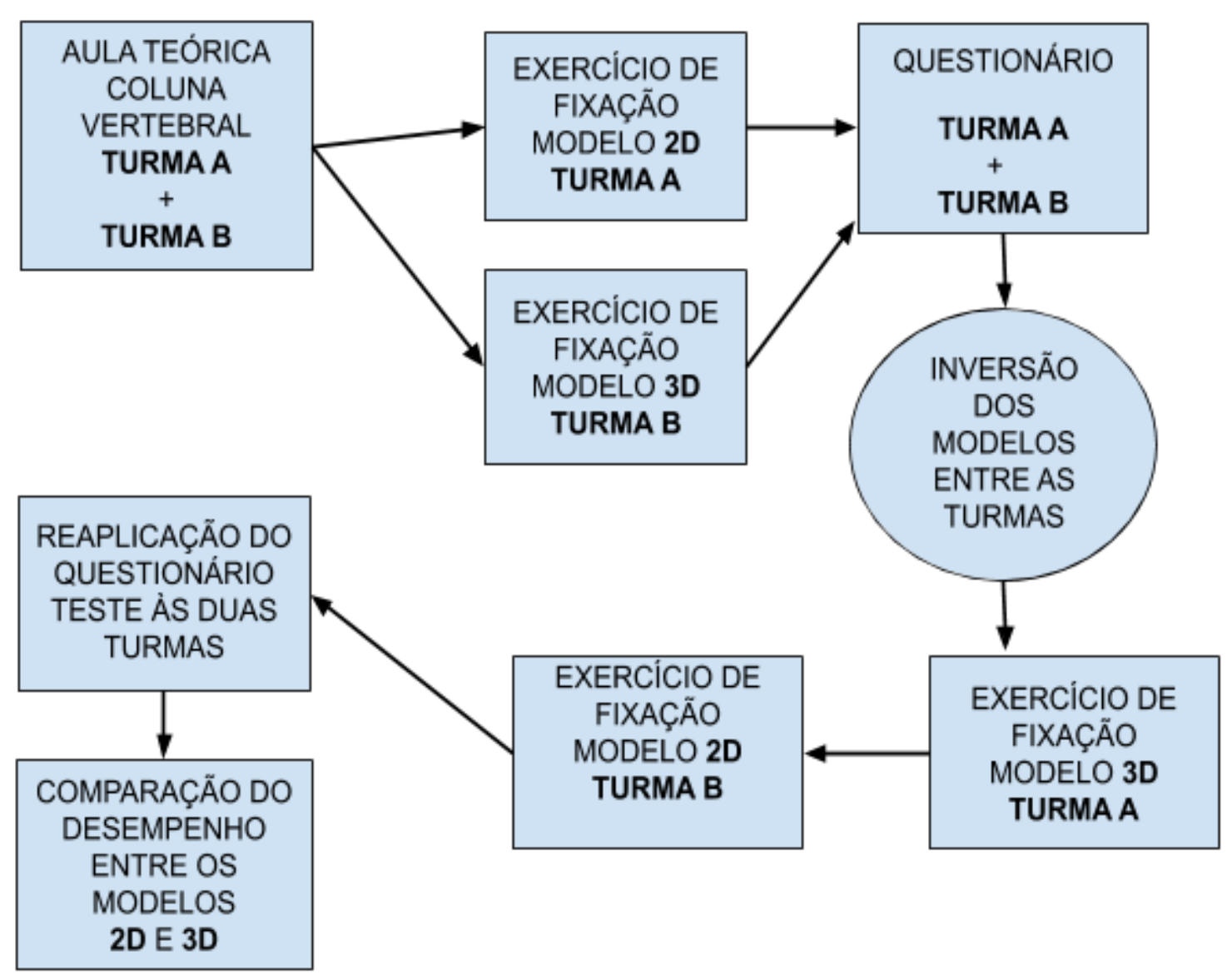

Figura 1 - Fluxograma de operacionalização da pesquisa

Após a aula teórica, ministrada pela professora responsável pela disciplina, com conteúdo sobre coluna vertebral humana, a turma "A" realizou exercícios de fixação, utilizando desenhos esquemáticos (2D) da vértebra lombar, nestes os discentes deveriam identificar os acidentes ósseos e colorir o desenho esquemático. A turma "B", realizou a mesma atividade, porém, utilizando o modelo vertebral lombar impresso em $3 \mathrm{D}$, seguindo o mesmo processo de identificação dos acidentes ósseos e colorindo as estruturas no modelo tridimensional.

Em ambos os exercícios foram apresentados os seguintes acidentes ósseos da vértebra lombar típica: corpo vertebral, forame vertebral, pedículo, processo transverso, processo articular, lâmina, processo espinhoso. Na Figura 2 (a) observa-se o modelo 2D utilizado (impresso na instituição), e (b) é visto o modelo 3D da vértebra lombar (Netter, 2015). 
(a)

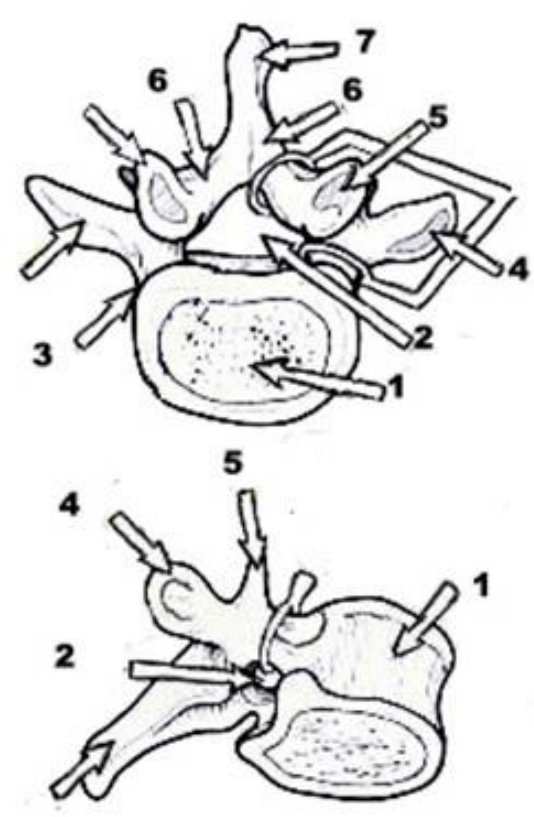

(b)

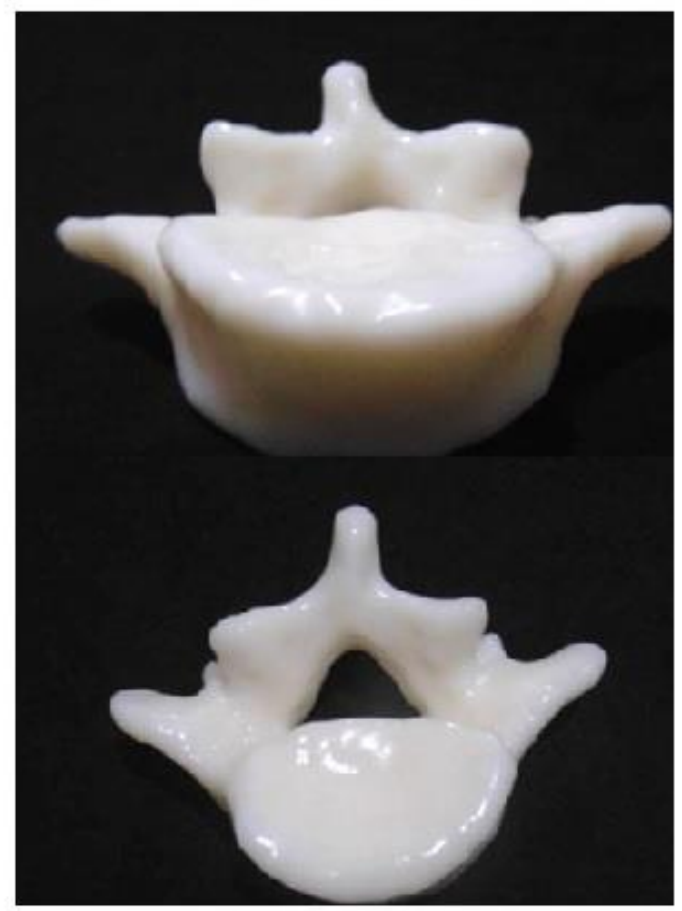

Figura 2 - Modelos 2D e 3D

Após os exercícios de fixação, os discentes foram encaminhados para o laboratório de anatomia, onde deveriam realizar a avaliação prática por meio de um questionário semiestruturado. A avaliação consiste em o aluno responder qual era o acidente ósseo sinalizado nas vertebra que estavam dispostas em bancadas, as quais em cada uma desta uma vértebra lombar (peça anatômica do cadáver) foi sinalizada com um acidente ósseo distinto, o discente deve observar e anotar a estrutura indicada, com duração de 1 minuto por acidente ósseo.

$\mathrm{Na}$ continuidade, houve uma inversão de recursos didáticos entre as turmas e o teste foi novamente aplicado. Destaca-se que ambos os grupos trabalharam tanto com o modelo 2D quanto com o modelo impresso em 3D, em seguida realizaram novamente o questionário semiestruturado.

\section{Resultados e discussões}

Para apresentação dos dados iniciais, relativos ao questionário, foram sistematizados os acertos por turma, antes e após a inversão de modelos.

Ao comparar o desempenho do grupo A, na aplicação das duas metodologias de ensino, o uso do modelo 3D mostrou-se superior ao esquema em 2D. Neste último recurso didático, o grupo apresentou maior dificuldade ao apontar o pedículo e o processo articular da vértebra lombar, enquanto com o modelo 3D não houve erros. Ressalta-se que o resultado obtido pode ser consequência da aplicação consecutiva de ambos os recursos didáticos, a qual houve o estudo com o recurso 2D e aplicação do questionário, em seguida estudo com o modelo 3D analisando os mesmos acidentes ósseos já estudados anteriormente e execução repetidamente da 
avaliação prática.

O mesmo fato ocorreu com o grupo B, onde o desempenho dos desenhos esquemáticos em $2 \mathrm{D}$ foi superior ao contrapor com o modelo $3 \mathrm{D}$, visto que o grupo iniciou os estudos com o modelo tridimensional e posteriormente com os desenhos esquemáticos. Ao realizar pela segunda vez o teste, o índice de acertos foi maior. Assim como a turma A, o grupo B também apresentou maior dificuldade ao identificar o processo articular na vértebra lombar.

Ressalta-se que a utilização de ambas as ferramentas de ensino se mostram complementares. O achado corrobora com pesquisa de Kimura (2019) que evidencia a importância da utilização de cores e imagens para a compreensão da nomenclatura e termos, bem como contribuem para a transposição do conhecimento teórico para o prático de forma lúdica. $\mathrm{O}$ mesmo ocorre com aplicação de peças tridimensionais no ensino, como confirma estudo de Albuquerque et al. (2020) que analisou o benefício do estímulo tátil de modelos sintéticos da anatomia cardíaca, de forma que possibilitou a transformação do discente como protagonista de sua própria aprendizagem, superando dificuldades inerentes ao ensino de anatomia.

A metodologia utilizada para o ato de ensinar precisa dinamizar o processo, despertar o interesse pelo aprendizado além de concretizar o conhecimento adquirido. Salbego et al. (2015) acredita que seja necessária uma reformulação da metodologia teórico-prática relacionada ao processo ensino-aprendizagem de anatomia humana, sendo importante envolver os discentes em discussões a respeito de quais maneiras podem ser mais efetivas na consolidação do conhecimento. Fundamentado no resultado da pesquisa, sugere-se que a prática de dois recursos didáticos de forma subsequente melhora os resultados avaliativos.

No Quadro 1 observa-se o total de acertos utilizando os dois recursos. Em ambos os recursos didáticos, o corpo vertebral e o forame vertebral, obtiveram $100 \%$ dos acertos, provavelmente pela facilidade em identificar visualmente os acidentes ósseos. O índice de acertos com os desenhos em 2D foi menor nas seguintes estruturas: pedículo (79\%) e processo articular (79\%). Já ao analisar o desempenho do recurso em 3D os acidentes ósseos com menor índice de acertos foram: pedículo (86\%), processo articular (82\%) e processo espinhoso (82\%).

\section{Quadro 1 - Total de acertos por modelo}

\begin{tabular}{lcc}
\hline Acidentes ósseos & $\begin{array}{c}\text { ACERTOS } \\
\text { 2D\% }\end{array}$ & $\begin{array}{c}\text { ACERTOS } \\
\text { 3D \% }\end{array}$ \\
forame vertebral & $100 \%$ & $100 \%$ \\
corpo vertebral & $100 \%$ & $100 \%$ \\
pedículo & $79 \%$ & $86 \%$ \\
processo transverso & $93 \%$ & $93 \%$ \\
processo articular & $79 \%$ & $93 \%$ \\
superior & & \\
lâmina & $93 \%$ & $93 \%$ \\
processo espinhoso & $93 \%$ & $82 \%$ \\
\hline
\end{tabular}

Fonte: Da Autora (2019)

O processo espinhoso foi o acidente ósseo com menor número de acertos com o modelo 3D, apesar deste ser bem destacado em peças anatômicas, o fato é contraditório, visto que espera-se que o contato físico com a peça, tecnicamente, 
contribuiria significativamente na assimilação de uma estrutura tão visualmente destacada. Dessa forma, a vantagem espacial deste recurso, descrita por Yammine e Violato (2015), em uma meta-análise que explora a eficácia de modelos físicos em 3D, não se confirmou em relação a esse acidente ósseo.

$\mathrm{O}$ pedículo foi um acidente ósseo que denotou dificuldade independentemente do modelo utilizado como recurso didático, porém sendo mais acentuada com o uso do recurso didático 2D. Justifica-se o fato pela sua localização anatômica, sendo uma área de ligação entre o processo transverso e o corpo vertebral, segmento este difícil de ser identificado e compreendido em um desenho esquemático.

Observa-se que não houve um rendimento impactante com o uso do modelo vertebral $3 \mathrm{D}$, como recurso didático, pois a porcentagem de erros, ficou de $8,19 \%$ contra $10,77 \%$ do modelo $2 \mathrm{D}$. Contudo, nota-se um desempenho superior com o uso do recurso 3D, pois o número de acertos por estruturas, no geral, foi maior. Abouhashem et al. (2015) afirma que reproduzir modelos tridimensionais de ossos mostra-se vantajoso devido a capacidade da técnica de impressão 3D replicar peças "com alto nível de precisão preservando os valores visuais e hápticos do modelo real". Desse modo, a vantagem do recurso didático 3D em relação ao modelo $2 \mathrm{D}$, verificada na presente pesquisa, pode estar realmente relacionada à facilidade de visualização dos acidentes ósseos em todos os ângulos da peça.

\section{Conclusão}

O avanço tecnológico vem beneficiando diversos setores da sociedade, assim como o meio educacional com o uso de ferramentas como a impressão 3D. Uma vez que a aplicação de recursos 3D no ensino de anatomia se mostrar expoente percebe-se a necessidade de formulação de metodologias que consigam usufruir dos instrumentos de forma dinâmica, incorporando variados recursos didáticos de aprendizagem juntamente a outros recursos existentes.

Considerando desempenho por turma, em que a Turma A apresentou resultado inferior realizando o primeiro contato com recurso $2 \mathrm{D}$ e resultado positivo ao realizar o questionário pela segunda vez, com uso do recurso $3 \mathrm{D}$, enquanto a Turma B apresentou resultado inferior ao utilizar no primeiro momento o recurso $3 \mathrm{D}$, e positivo ao utilizar no segundo momento o recurso $2 \mathrm{D}$, supõe-se que: uma segunda oportunidade de realização da avaliação, envolvendo a mesma ordem de apresentação das estruturas facilitou a identificação dos acidentes ósseos.

Apesar de não apresentar resultados impactantes ao comparar o desempenho do modelo 3D com o desenho esquemático em $2 \mathrm{D}$, ainda assim afirmase que a impressão tridimensional de estruturas anatômicas permite o contato físico e possibilita um processo de ensino aprendizagem prático.

Percebeu-se que para possibilitar um eficiente processo de ensino aprendizagem faz-se necessário a associação de diferentes recursos. Dessa forma, concluiu-se que os modelos vertebrais lombares em 2D e 3D são complementares, sendo, interessante aplicar metodologias que aliem o uso dos dois modelos.

Tendo em vista que objetivo desse estudo foi realizar uma análise do uso de modelos vertebrais $2 \mathrm{D}$ e $3 \mathrm{D}$ como recurso didático para o processo de ensinoaprendizagem, acredita-se que, um próximo passo seria um aprofundamento em posteriores pesquisas, nas quais a utilização de uma abordagem qualitativa, captando com maior riqueza de detalhes a percepção dos alunos, possibilitaria um avanço nas V. $18 \mathrm{~N}^{\circ}$ 1, julho, 2020 DOI: RENOTE 
reflexões sobre pertinência e eficácia dos recursos no contexto de ensinoaprendizagem.

\section{Referências Bibliográficas}

ABOUHASHEM, Yousef et al. The application of 3D printing in anatomy education. 2015. Disponível em: 〈http://creativecommons.org/licenses/by/4.0/>. Acesso em: 12 mar. 2019.

ALBUQUERQUE, José Danilo dos Santos et al. Aprendendo de olhos fechados: ensino de anatomia do coração e vasos da base por meio de identificação tátil.

Revista Eletrônica Acervo Saúde, João Pessoa, v. 49, p. 1-7, jun. 2020. Disponível em: <https://doi.org/10.25248/reas.e3349.2020>. Acesso em: 09 jul. 2020.

CORREDERA, Blanca Mompéo. Metodologias y materiales para el Aprendizaje de la Anatomía Humana: Percepciones de los Estudiantes de Medicina 'nativos digitales'. FEM (Ed. impresa), Barcelona, v. 17, n.2, p.99-104, jun. 2014. Disponível em: <http://scielo.isciii.es/scielo.php?script=sci_arttext\&pid=S201498322014000200007>. Acesso em: 21 set. 2018.

ESTAI, Mohamed; BUNT, Stuart. Best teaching practices in anatomy education: A critical review. Annals of anatomy, n. 208, p. 151-157. 2016. Disponível em: <https://www.sciencedirect.com/science/article/abs/pii/S0940960216300322>. Acesso em: 18 set. 2018.

GRANDE NETO, Newton Salvador. Utilização de material alternativo para obtenção e caracterização de biomodelos, por meio da técnica de impressão 3D printer. 2016. 33 f. Dissertação de Mestre em Engenharia Mecânica - Faculdade de Engenharia - UNESP, São Paulo, 2016.

KIMURA, Luciana Akemi. Produção e avaliação de pranchas com imagens para colorir no ensino de biologia. 2019. 74 f. Dissertação de Mestre em Enisno de Biologia - Universidade Estadual de Campinas, Instituto de Biologia, São Paulo, 2019.

MATOZINHOS, Isabela Penido et al. 3D Impressão 3D: inovações no campo da medicina. Revista Interdisciplinar Ciências Médicas, v. 1, n. 1, p. 143-162. 2017. Disponível em: <http://revista.fcmmg.br/ojs/index.php/ricm/article/view/14>. Acesso em: 27 out. 2018.

NETTER, Frank H. Atlas de anatomia humana. 6. ed. São Paulo: Elsevier, 2015. NISHIMURA, Paula Lumi Goulart et al. Prototipagem rápida: um comparativo entre uma tecnologia aditiva e uma subtrativa. In: $\mathbf{1 2}^{\circ}$ Congresso Brasileiro de Pesquisa e Desenvolvimento em Design, v. 2, n. 9, p. 4481-4491, 2016, Belo Horizonte. Anais. Belo Horizonte: Bucher Desing Proceedings.

PAIVA, Lilian R Mendes et al. Aplicação para dispositivos móveis utilizando tecnologias interativas: a Realidade Virtual e Aumentada aplicada ao estudo da anatomia humana. In: X Encontro Anual de Computação, 2013, Goiás. Anais. Catalão: ENACOMP 2013.

SALBEGO, Cléton et al. Percepções acadêmicas sobre o ensino e a aprendizagem em anatomia humana. Rev. bras. edu. med., Rio de Janeiro, v. 39, n. 1, p. 23-31, mar. 2015. Disponível em:

<http://www.scielo.br/scielo.php?script=sci_arttext\&pid=S010055022015000100023\&lng=en\&nrm=iso>. Acesso em: 08 jul. 2020.

SILVA, Joab Júlio de Souza; MALAGÓN, Luiz Arturo Gómes. Fabricação de modelos anatômicos utilizando prototipagem rápida. Revista de Engenharia e V. $18 \mathrm{~N}^{\mathrm{o}} 1$, julho, 2020 RENOTE DOI: 
Pesquisa Aplicada. Recife, v. 2, n. 1, p. 613-621, 2016. Disponível em:<http://revistas.poli.br/index.php/repa/article/view/523>. Acesso em: 21 out. 2018.

YAMMINE, Kaissar; VIOLATO, Claudio. A meta analysis of the educational effectiveness of three dimensional visualization Technologies in teaching anatomy. Anatomical Sciences Education. Nova Jersey, v. 8, n. 6, p. 525-538. dez. 2014. Disponível em: < https://pubmed.ncbi.nlm.nih.gov/25557582/>. Acesso em: 21 maio 2019. 